\title{
Kernos
}

Revue internationale et pluridisciplinaire de religion grecque antique

$4 \mid 1991$

Varia

\section{Dionysos chez Homère ou la folie divine}

\section{Paul Wathelet}

\section{OpenEdition}

Journals

Édition électronique

URL : http://journals.openedition.org/kernos/286

DOI : $10.4000 /$ kernos.286

ISSN : 2034-7871

Éditeur

Centre international d'étude de la religion grecque antique

Édition imprimée

Date de publication : 1 janvier 1991

Pagination : 61-82

ISSN : 0776-3824

Référence électronique

Paul Wathelet, "Dionysos chez Homère ou la folie divine », Kernos [En ligne], 4| 1991, mis en ligne le

11 mars 2011, consulté le 30 avril 2019. URL : http://journals.openedition.org/kernos/286 ; DOI :

10.4000/kernos.286

Kernos 
Kernos, 4 (1991), p. 61-82.

\section{DIONYSOS CHEZ HOMÈRE OU LA FOLIE DIVINE}

Dionysos occupe dans l'épopée homérique et dans l'œuvre d'Hésiode une place très discrète. On s'efforcera dans les pages qui suivent de trouver les raisons de cette discrétion et de voir dans quelle mesure le Dionysos de l'époque d'Homère correspond à la figure classique du dieu.

Depuis longtemps des spécialistes avaient conclu, avec quelque imprudence, que le peu d'importance du dieu dans l'épopée était dû à son entrée tardive dans le panthéon hellénique ${ }^{1}$. Dionysos serait un dieu étranger, sans doute thrace et dont le succès ne se serait confirmé qu'après Homère. Dans l'histoire de la religion grecque, on se méfiera d'une méthode qui consiste à s'interroger sur l'origine de chaque divinité avant de s'attacher à définir sa personnalité ${ }^{2}$. Les principaux dieux grecs sont facilement polyvalents. Trouver l'origine de tel trait propre à un dieu - en admettant que la chose soit possible - n'offre de

1 K.O. MÜLLER, Orchomenos und die Minyer, Breslau, 18442, p. 374-377; C.A. LOBECK, Aglaophamus, Königsberg, 1829, I, p. 289-298; et, encore récemment, A.J. Festugière, Les Mystères de Dionysos, in RB, 44 (1935), p. 192-211 [= Etudes de religion grecque et hellénistique, 1972, p. 13-63] et M.L. FREYBURGERGalland, Sectes religieuses à Rome, in M.L. Freyburger-Galland - G. FREYBURGer - J.C. TAUTIL, Sectes religieuses en Grèce et à Rome, Paris, Les Belles Lettres, 1986 (Realia), p. 38-40. Pour l'historique du problème, cf. W. BURKERT, Greek Religion, Harvard, 1985, p. 161-167. L'origine thrace du dieu paraît trouver un appui en $\mathrm{Z} 133$ dans la mesure où le Nvớlov a été placé en Thrace par la tradition. Le trait mythique qui montre le dieu toujours en mouvement (M. Detienne, Dionysos à ciel ouvert, Paris, Hachette, 1986, p. 1520) a été interprété par certains Modernes comme l'indice de son arrivée tardive dans le panthéon grec.

2 Nombreux sont les travaux qui s'interrogent par exemple sur l'origine d'Apollon, sans s'efforcer d'abord de définir la personnalité du dieu. Le cas de Dionysos est parallèle à celui d'Arès que l'on a dit arrivé tard dans le panthéon grec et d'origine thrace, jusqu'au jour où son nom est apparu sur les tablettes en linéaire $\mathrm{B}$. 
toute manière qu'une parcelle de réponse à la question complexe de l'origine du dieu en question ${ }^{3}$.

Quoi qu'il en soit, le déchiffrement du linéaire $B$ a, sur le point qui nous occupe aussi, ouvert des perspectives nouvelles, puisque le nom de Dionysos paraît, bien être attesté en mycénien ${ }^{4}$. Ainsi, même s'il est de provenance étrangère, Dionysos est arrivé en Grèce à une époque antérieure à celle où s'est forgée la tradition épique. On ne peut invoquer, en la matière, le caractère difficilement explicable par le grec, du théonyme $\mathrm{e}^{5}$ : la majorité des noms de dieux helléniques se trouvent dans le même cas.

Dionysos est mentionné en huit passages de l'épopée ${ }^{6}$. Quatre d'entre eux présentent un point commun; il s'agit d'une brève allusion à une légende relative au dieu : deux concernent sa naissance et deux son intervention dans la légende d'Ariane.

Au chant XIV de l'Iliade, Héra fait tout pour séduire son mari et Zeus se laisse prendre. En fait, les bonnes dispositions d'Héra ne constituent qu'une feinte pour endormir Zeus et le détourner de venir en aide aux Troyens. La majestueuse hiérogamie du Ciel et de la Terre est ramenée à une ruse grossière ${ }^{7}$. Quoi qu'il en soit, Zeus, attiré par la beauté de sa femme, lui tient un discours qui, en d'autres circonstances, la mettrait hors d'elle. «Jamais», dit-il, «je n'ai éprouvé un pareil désir pour ...» et il énumère une série de ses nombreuses bonnes fortunes, dont Sémélé qui mit au monde Dionysos. On peut ne pas apprécier le sens des conve-

Le problème peut être discuté à perte de vue, mais il semble bien que les Grecs avaient une certaine conscience de la personnalité au moins des divinités principales. L'épopée homérique a beaucoup contribué à définir et à fixer le caractère des dieux (comme semble l'avoir senti HÉroDOTE, II, 53, 2). Certaines épithètes cultuelles paraissent avoir conservé des traces d'une situation où une divinité peut prendre des caractères d'une autre. C'est peut-être le cas d'HéraAphrodite, honorée à Sparte (PAUS., III, 13, 9).

4 M.S. RuIPEREz, The Mycenaean Name of Dionysos, in Res Mycenaeae. Akten des VII. Internationalen Mykenologischen Colloquiums in Nürnberg vom 6.-10. April 1981, Göttingen, 1983, p. 408-412. W. BURKERT, Greek Religion, p. 31, signale de surcroît que le culte très ancien à Ayia Irini, à Céos, se révèle ultérieurement être un culte de Dionysos.

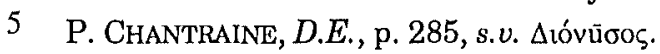

6 Z 130-131; $\Xi$ 325; $\lambda$ 321-325; $\omega$ 74; Théog., 940-942, 947-949; Trav; 614; Bouclier,

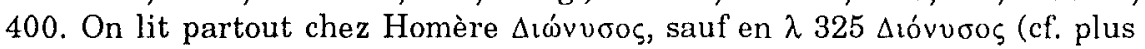
haut, n. 4 et 5).

7 Sur l'ensemble de ce passage, cf. P. WATHELET, Homère. Du Mythe à la mythologie, in Actes du Colloque de Liège et Louvain-la-Neuve 1981, Louvainla-Neuve, 1983, p. 210-211. 
nances qui se manifeste chez Zeus, mais l'aède se repose un instant en énumérant un Catalogue des femmes du Cronide, un de ces multiples catalogues que les chanteurs devaient connaittre par cœur afin de fixer leur répertoire et de n'être jamais embarrassés dans leur œuvre de composition. Il s'agit de toute une série de légendes bien connues du public des connaisseurs et dont la seule évocation lui suffit. Point n'est besoin de présenter les héroïnes ou de rappeler leurs aventures, les auditeurs, qui sont au fait des récits épiques, sont au courant. On peut en conclure, de la présence de Sémélé et de Dionysos dans ce passage, que le dieu et sa légende sont largement connus en Grèce à cette époque.

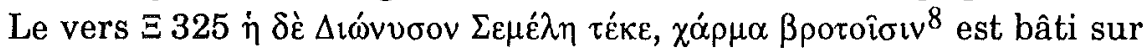
le même schéma que, dans la Théogonie hésiodique, le premier de trois vers consacrés au même sujet :

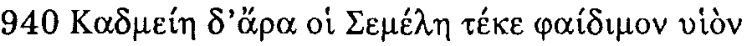

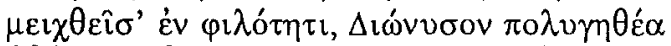

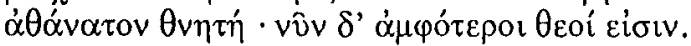

Les trois vers se trouvent dans le Catalogue des Hérö̈nes qui termine le poème. Après avoir évoqué Maia, mère d'Hermès par les œuvres de Zeus, le poète mentionne Sémélé. Le passage contient un certain nombre d'expressions traditionnelles ou dérivées de la tradition: $\varphi \alpha i \delta$ ¿ $\mu$ ov viòv, $\mu \varepsilon \imath \chi \varepsilon \varepsilon \hat{\imath} \sigma^{\prime} \dot{\varepsilon} v \varphi \imath \lambda o^{\prime} \tau \tau \imath$ et $\dot{\alpha} \theta \dot{\alpha} v \alpha \tau o v \theta v \eta \tau \eta$. Cette dernière formule, en asyndète, souligne une particularité de Dionysos : être divin, il est fils d'une mortelle. Les autres dieux grecs importants descendent de parents qui sont tous deux des immortels ${ }^{9}$. Certes, Héraklès est fils d'Alcmène,

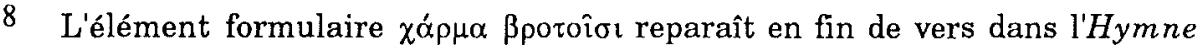
pseudo-homérique à Apollon (25), où il est appliqué au fils de Léto. L'expression ne semble pas très ancienne dans la tradition à cause de la correptio attica indispensable à la scansion dans $\beta$ potoî́ı. P. WATHELET, La coupe syllabique et les liquides voyelles dans la tradition formulaire de l'épopée grecque, in Recherches linguistiques en Belgique, 1966, p. 166-168. Le terme $\chi \alpha \dot{\rho} \mu \alpha$ est encore lié à Dionysos dans le Bouclier pseudo-hésiodique (400, cf. aussi fr. 239 , 1 Merkelbach-West).

9 M. Detienne, Dionysos à ciel ouvert, p. 24-25. Cf. W. Burkert, Greek Religion, p. 128-129, qui note que les enfants de Zeus, nés de femmes mortelles sont en général mortels, sauf Hélène et Pollux. Sans se prononcer ici sur le statut de ces personnages à date très ancienne, on notera que, pour les Grecs de l'époque d'Homère et pour ceux de l'époque classique, il s'agit d'êtres qui, tel Héraklès, ont connu une vie humaine, avant de passer à l'immortalité. Ce n'est pas le cas de Dionysos, dieu dès sa naissance. La légende post-homérique expliquait que Sémélé avait été embrasée par son divin amant et que celui-ci avait sauvé le jeune Dionysos encore à naître en l'enfermant dans sa propre cuisse et en lui conférant par là-même l'immortalité (cf. W. OTTO, Dionysos. Le 
mais il n'atteindra à l'immortalité qu'après sa mort. Il y a là, pour Dionysos, un trait qui brouille les hiérarchies reconnues et qui souligne le rôle de l'humanité dans l'apparition du dieu.

Deux autres mentions, l'une dans l'Odyssée et l'autre dans la Théogonie, doivent être examinées ensemble : elles figurent aussi dans des Catalogues et elles traitent du même sujet, mais avec des versions divergentes. La célèbre Nékyia de l'Odyssée montre Ulysse venu à l'entrée des Enfers pour consulter le devin Tirésias. Toujours curieux, le fils de Laërte en profite pour voir le monde des morts. C'est, de nouveau, l'occasion pour Homère de placer un Catalogue des héroïnes. Parmi celles-ci se trouve Ariane, la fille de Minos :

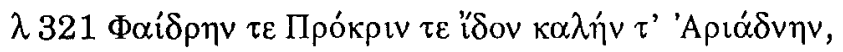

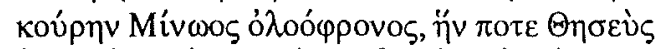

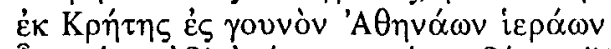

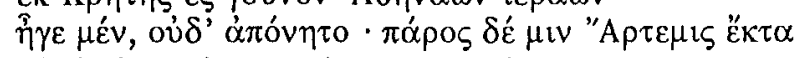

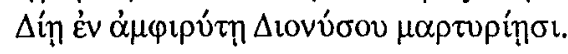

Quelques vers après ceux qui ont été évoqués plus haut et toujours dans le même Catalogue des héroïnes, la Théogonie d'Hésiode fait allusion, elle aussi, à la légende d'Ariane

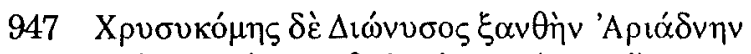

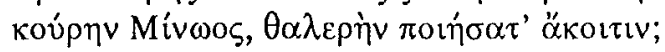

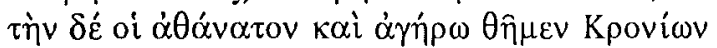

Les deux passages comportent un certain nombre d'éléments traditionnels ${ }^{10}$. Ariane semble une héroïne très ancienne, liée à la Crète minoenne ${ }^{11}$. L'Iliade ( $\Sigma$ 591) rapporte que Dédale avait construit pour Ariane une aire de danse. De telles aires de danse avaient manifestement une valeur religieuse à l'époque minoenne ou mycénienne ${ }^{12}$. Ariane est peut-être une ancienne déesse, dont le nom 'Aptód $\delta \eta \eta$ serait

mythe et le culte, trad. de l'all. par P. Lévy, Paris, 1969, p. 71-80). Le cas d'Asklépios est parallèle, mais il semble avoir été considéré d'abord comme un héros (W. BURKERT, Greek Religion, p. 214).

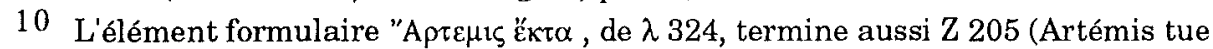

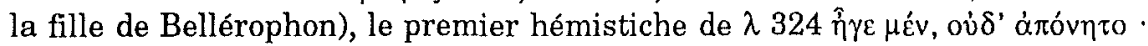

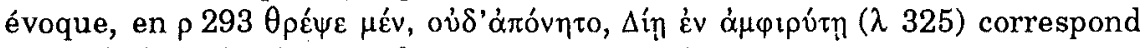

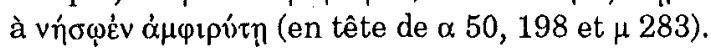

11 Sur Ariane, cf. SToll, art. Ariadne, in W.H. Roscher, Lexikon ..., I, c. 540-546, et M.-L. Bernhard - W.A. DAszeWsKI, art. Ariadne, in LIMC, III, p. 1050-1070; W.F. OTTo, Dionysos ..., p. 190-197.

12 W. BuRKERT, Greek Religion, p. 34. 
une forme crétoise de $\alpha \rho^{\prime} \alpha \dot{\gamma} \gamma \vee \eta$, «la très sainte» ${ }^{13}$. Fille de Minos, la légende rapporte qu'elle s'était éprise de Thésée et qu'elle l'avait aidé à sortir du labyrinthe. Le passage par le labyrinthe est une manière de descente aux Enfers ou, au moins, un parcours initiatique ${ }^{14}$. L'appui qu'elle avait apporté à Thésée n'est pas sans rappeler l'assistance qu'Athéna a fournie à plusieurs héros, dont spécialement Ulysse. Toutefois, à l'inverse d'Athéna, Ariane est guidée par l'amour. Tant dans son mythe que dans les représentations que l'Antiquité nous a laissées, l'héroïne présente un côté érotique, elle est très proche d'Aphrodite a laquelle elle est parfois associée ${ }^{15}$.

Enlevée par Thésée, Ariane est ensuite abandonnée dans l'île mystérieuse de Diè d'après Homère ${ }^{16}$, à Naxos d'après les sources plus tardives. Dionysos la trouve, il l'emmène et l'épouse. Homère, toutefois, se fait l'écho d'un autre récit : Ariane est tuée par Artémis "au témoignage de Dionysos" 17 .

Les raisons de l'abandon d'Ariane par Thésée sont diverses selon les auteurs, mais certaines sources invoquent l'intervention d'Athéna ${ }^{18}$, qui oblige Thésée, son protégé, à abandonner sa conquête. Athéna ne peut rien avoir de commun avec une créature proche d'Aphrodite. Ariane, liée à la déesse des passions amoureuses incontrôlées, était, par le fait même, proche de Dionysos, dieu irrationnel et impulsif. Rien d'étonnant dès lors qu'ils se soient rencontrés et que Dionysos ait épousé Ariane. La version de l'Odyssée vient jeter une

13 Sur son culte, L.R. FARNeLL, Greek Hero Cults ..., Oxford, 1921, p. 48-49, et The Cults of the Greek States, Oxford, 1896, II, p. 631-635. Ailleurs qu'en $\Sigma 592$ où il désigne Ariane, l'adjectif $\kappa \alpha \lambda \lambda\left(\pi \lambda{ }^{\prime} \kappa \alpha \mu \rho_{\zeta}\right.$ est toujours appliqué chez HOMÈRE à des déesses : Déméter ( $\Xi 326)$, Thétis ( $\Sigma$ 407, Y 207), Léto (H. Apollon, 101) et

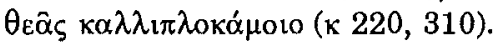

14 M. ELIADE, Initiation, rites, sociétés secrètes. Naissances mystiques. Essai sur quelques types d'initiation, Paris, 1959, p. 133-139.

15 Le caractère érotique de l'héroïne est souligné par PHILOchore, $328 \mathrm{~F} \mathrm{17a}$ Jacoby, cité par Plutarque (Thésée, 16, 6). Cf. W. OTto, Dionysos, p. 190-197. L'auteur écarte l'opinion selon laquelle Ariane était une déesse, mais il souligne que l'héroïne était très proche d'Aphrodite. H. Jeanmaire, Couroi et Courètes, p. 319 .

16 Plusieurs petites îles de l'Égée sont appelées «Dia», féminin de l'adjectif dérivé de Sîoç. E. MEYer, art. Dia 4, in Der Kleine Pauly, I, c. 1504. - L'assimilation de Dia à Naxos est attestée chez CALLIMAQUE, fr. 601 Pfeiffer.

17 Le terme $\mu \alpha \rho$ tupín n'apparaît qu'ici chez HомÈRE.

18 PhÉRÉcyde d'Athènes, $3 \mathrm{~F} 148$ Jacoby, cité par Schol. MV à $\lambda$ 322. ThÉocrite (II, 45-46), parle simplement d'un oubli de Thésée. Sur diverses versions, cf. PluT., Thésée, 20. 
ombre curieuse sur la fin idyllique de l'aventure : Ariane est tuée par Artémis à cause du témoignage de Dionysos. L'expression isolée

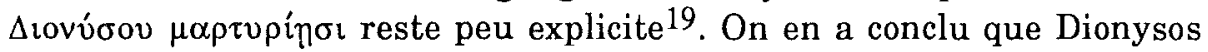
avait épousé Ariane avant sa liaison avec Thésée et que le dieu s'était vengé. Artémis apparaît comme la gardienne attitrée de la féminité et, dans l'épopée, elle frappe de mort brutale des femmes qui sont sorties de leur condition, comme la mère d'Andromaque, qui régnait à Thèbessous-le-Plakos ${ }^{20}$. Ariane a trahi son père et son pays. Est-elle par son action sortie de son rôle féminin?

Même si elle est devenue l'épouse de Dionysos et si, d'après la tradition, elle lui aurait donné des enfants ${ }^{21}$, elle n'est pas immortalisée pour la cause et plusieurs sources indiquent qu'elle est morte. Cette mort n'est guère compatible avec l'hypothèse d'une Ariane qui aurait été une déesse à date ancienne, à moins que nous ne soyons en présence d'une déesse qui meurt pour renaître ultérieurement.

La mention de la légende d'Ariane dans l'épopée apporte au moins trois enseignements : dieu des poussées impulsives et irraisonnées, Dionysos est associé à Ariane, elle-même proche d'Aphrodite. Ariane semble être une héroïne très ancienne, minoenne sans doute et le dieu apparaît à ses côtés. Suivant la version homérique, Dionysos cause la mort d'Ariane, il ne peut se stabiliser dans l'institution du mariage. De plus, dieu ambigu, il donne à la fois la vie et la mort.

Trois autres allusions doivent être examinées ensemble : il s'agit des cadeaux faits par Dionysos. Dans les Travaux, le dieu donne la vie qui fait naître la joie :

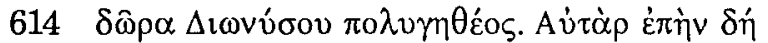

Le génitif en -ov du théonyme est irréductible en -o-o et, partant, relativement récent dans la tradition épique ${ }^{22}$. En revanche, l'adjectif $\pi \circ \lambda v \gamma \eta \theta n$ s est déjà apparu, appliqué au dieu, dans la Théogonie (941). À la fin de l'Odyssée, on trouve une allusion à un présent de Dionysos,

19 Sur l'explication qui suggère qu'Ariane était déjà mariée à Dionysos quand Thésée l'emmena, cf. M.L. WeST, Hesiod Theogony, p. 418.

20 P. Wathelet, Dictionnaire des Troyens de l'Iliade, Université de Liège,

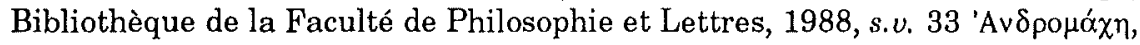
p. 282.

21 Sur les enfants d'Ariane et de Dionysos, cf. Plut., Thésée, 20, 8; Ps.-Apollod., Epitome, I, 9.

22 C.J. RUIJGH, La morphologie du grec, in SMEA, 20 (1979), p. 72-74. 
mais, ici, ce présent a un usage funèbre. C'est lui qui a donné une amphore d'or à Thétis et la déesse y enfermera les cendres de Patrocle, d'Achille et d'Antiloque ${ }^{23}$ :

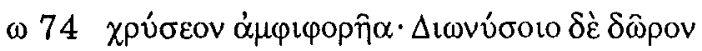

D'un côté, Dionysos donne la joie; de l'autre, il est lié à la mort. Un vers du Bouclier pseudo-hésiodique fait écho à cette situation :

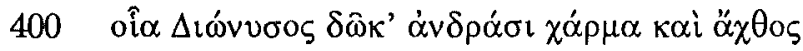

c'est une allusion aux effets tantôt positifs, tantôt négatifs du vin. Le dernier vers reprend le terme de $\chi \alpha ́$ ó $\rho \alpha$ qui était déjà apparu précédemment en $\Xi 325$, cette fois pour désigner le dieu lui-même.

La raison pour laquelle Dionysos a donné une amphore d'or à Thétis apparaîtra à la lumière du seul récit un peu détaillé que l'Iliade fournit au sujet du dieu. Comme on va le voir, il avait contracté une dette vis-àvis de la mère d'Achille.

Le seul développement de quelque longueur relatif à Dionysos figure au chant VI de l'Iliade. Homère y a placé la rencontre de Glaukos et de Diomède. Tout au début de la scène, Diomède interroge Glaukos sur son identité. Il ne voudrait pas combattre un immortel sans le savoir, car ce genre de combats, auxquels pourtant il vient de s'adonner, comporte un grave danger. Diomède est un héros que l'hybris ne cesse de menacer, l'hybris suprême serait pour lui de s'emparer du char solaire, gage sans doute d'immortalité, ainsi qu'il apparaît dans la Dolonie ${ }^{24}$. Le piquant est que l'adversaire qu'il est sur le point de combattre, Glaukos, est originellement lui-même un immortel ${ }^{25}$.

Les exemples sont nombreux dans la mythologie qui sont destinés à décourager les actes d'hybris. Le récit qui vient aux lèvres de Diomède à l'appui de son inquiétude, c'est l'histoire de Lycurgue, fils de Dryas :

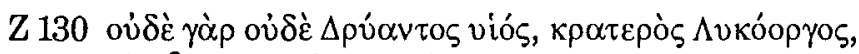

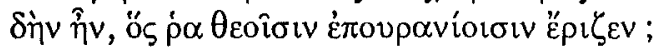

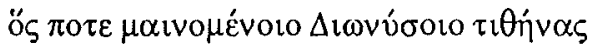

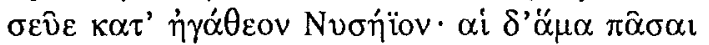

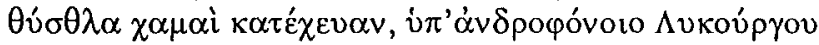

23 Antiloque est proche d'Achille. C'est lui qui est chargé de lui annoncer la mort de Patrocle (P 684-699 et $\Sigma$ 1-33). Sur le caractère ambigu du vin et du dieu auquel il est lié, cf. M. Detienne, Dionysos à ciel ouvert, p. 54-58.

24 P. WATHELET, Rhésos ou la quête de l'immortalité, in Kernos, 2 (1989), p. 213 231.

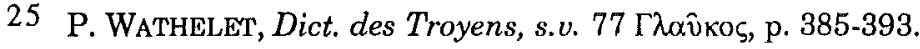




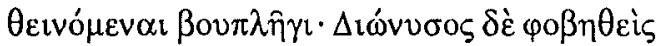

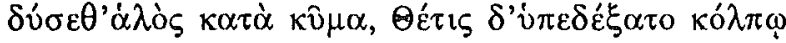

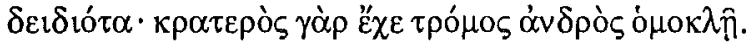

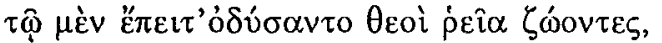

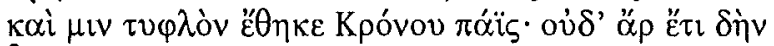

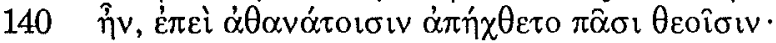

Récit exemplaire qui prend soin d'opposer très fortement les dieux et les hommes; or, du côté des dieux, se trouve Dionysos. Lycurgue porte un nom grec bien connu; c'est "celui qui écarte les loups» 26 . Le loup joue un rôle important dans la mythologie grecque ${ }^{27}$. Animal sauvage, qui vit en bande, il connaît néanmoins des règles de vie en société bien établies. Il symbolise le monde des marginaux et des jeunes gens en voie d'initiation, qui, s'ils réussissent les épreuves de passage, pourront être membres à part entière du groupe des adultes. En attendant que leur initiation soit terminée, les jeunes gens vivent en marginaux, en loups, sous le patronage d'Apollon. Ils constituent donc un danger pour la société qui se défend contre eux. C'est bien ce que fait Lycurgue, "celui qui écarte les loups». Dans la mesure où leur légende est connue, les autres Lycurgue de la mythologie semblent être dans le même cas ${ }^{28}$. Outre notre héros, le nom désigne un héros primordial arcadien, c'est lui qui, d'après l'Iliade (H 142-146), avait tué Aréithoos, sorte de sauvage armé d'une massue ${ }^{29}$. Un autre Lycurgue fut prêtre de Zeus à Némée, il fut le père d'Opheltès, un enfant royal dont la mort a été à l'origine des jeux néméens ${ }^{30}$. Les autres Lycurgue sont des personnages secondaires, placés dans des énumérations de personnages : un fils $\mathrm{d}^{\prime} H$ éraklès ${ }^{31}$ et un prétendant malheureux d'Hippodamie ${ }^{32}$. Un héros

26 H. von KAMPTZ, Homerische Personennamen, Göttingen, 1982 [1958], § 22 a 1,

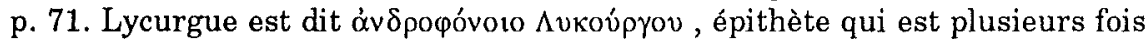
attribuée à Hector dans la formule "Екторо $840, \mathrm{P} 428,616, \Sigma 149, \Omega 509$, ce qui paraît avoir échappé à M. DetienNe, Dionysos à ciel ouvert, p. 106, n. 48). Le fait d'être "tueur d'hommes" n'a pas nécessairement, dans le monde épique, une coloration péjorative.

27 M. Detienne - J. Svenbro, Les loups au festin ou la Cité impossible, in M. DETIENNE - J.-P. VERNANT, La cuisine du sacrifice, p. 226.

28 DREXLER, art. Lykurgos, in W.H. RosCHER, Lexikon, II, c. 2191-2205.

29 H. von Geisau, art. Areithoos, in Der Kleine Pauly, I, c. 525; A. Severyns, Curiosités biographiques dans les poèmes homériques, in BAB, 34 (1948), p. 430.

30 Ps.-APOLLOD, Bibl., I, 9, 14; III, 6, 4.

31 Ps.-APOLLOD., Bibl., II, 7, 8.

32 PAUS., VI, 21, 10. 
doit être mis à part : il s'agit du législateur de Sparte qui semble, lui aussi, un personnage mythique ${ }^{33}$, et dont le père est tantôt Eunomos, tantôt Eukosmos. Dans les deux cas, l'anthroponyme est parlant ${ }^{34}$.

Notre Lycurgue est fils de Dryas, dont le nom est tiré de $\delta \rho \hat{\jmath} \varsigma$, l'arbre, le chêne ${ }^{35}$. L'anthroponyme est porté par plusieurs héros du nord de la Grèce, notamment un Lapithe, ami de Peirithoos, qui combattit les Centaures ${ }^{36}$, ou un fils d'Arès qui participa à la chasse au sanglier de Calydon ${ }^{37}$. On est donc en présence de personnages opposés à la sauvagerie, ce qui rejoindrait le caractère du Lycurgue qui nous occupe. Le nom aurait encore été donné au fils du même Lycurgue. Rendu fou par Dionysos, Lycurgue prit son fils pour une vigne et lui coupa les extrémités. Cette terrible histoire n'est pas rapportée par l'épopée, elle figure dans la tradition ultérieure: on y reviendra ${ }^{38}$.

Lycurgue se précipite sur le Nyseion ${ }^{39}$. Peu importe sa situation qu'on a cherchée dans de nombreux endroits. Il s'agit du domaine propre de Dionysos, peut-être au prix d'une étymologie populaire qui rapproche Nvonỉiov du second élément de $\Delta$ tóvvoos. Le domaine est qualifié de très saint. Y pénétrer pour un mortel constitue sans doute déjà une impiété. Mais Lycurgue ne s'est pas arrêté là, il a poursuivi les nourrices de Dionysos. La mention de nourrices implique que l'on a affaire à un bébé, le pluriel suggère un personnage extraordinaire ou par sa qualité ou par sa voracité. On apprendra, quelques vers plus loin, que Dionysos épouvanté s'est jeté dans la mer. Il s'agit donc, non plus d'un bébé allaité, mais d'un être plus âgé, capable de se déplacer par luimême. De toute façon, les nourrices ont été négligentes, puisqu'elles n'ont pas surveillé l'enfant dont elles avaient la charge et le dieu luimême, loin de se défendre ou de les défendre, ainsi qu'on aurait pu l'attendre d'une divinité, est effrayé et il s'enfuit. Le poète souligne la

33 K. KINZL, art. 4 Lykurgos, in Der Kleine Pauly, III, c. 823-824; J.T. HоOKer, The Ancient Spartans, Londres, 1980, p. 126-130.

34 A. FICK - F. BECHTEL, Die griechischen Personennamen, Göttingen, 18942,

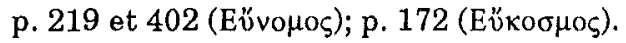

35 H. vON KAMPTZ, Homerische Personennamen, p. 168, $\S 63$ c, et p. $277, \S 76$ a.

36 A 263; Bouclier, 179.

37 Ps.-Apollod., Bibl., I, 8, 2; HyGIN, Fab., 45.

38 Ps.-Apollod., Bibl., III, 5, 1; Hygin, Fab., 132.

39 Fr. F. Schwarz, art. Nysa 2, in Der Kleine Pauly, IV, c. 217-218. Une plaine

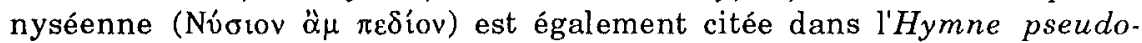
homérique à Déméter (17). C'est là que Koré jouait avec les Océanides, ce qui implique que cette plaine est proche de la mer : A. MotTe, Prairies et Jardins de la Grèce Antique. De la Religion à la Philosophie, Bruxelles, 1973, p. 166-167. 
peur de Dionysos : il est $\varphi \circ \beta \eta \theta \varepsilon i \varsigma, \delta \varepsilon \imath \delta$ เó $\tau \alpha$, le $\tau \rho o ́ \mu o \varsigma$ le tient. Contrairement aux autres dieux, Dionysos est incapable de manifester sa puissance. Tous les détails qui viennent d'être mentionnés donnent une impression d'étrangeté qui est confirmée par une précision :

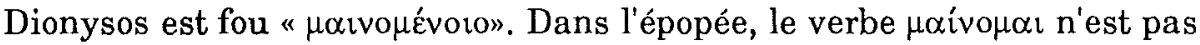
rare. Il est spécialement appliqué à Arès, dieu peu estimé, considéré comme un étranger et dont la folie sanguinaire est connue (E 831, O 128, $\mathrm{E} 717, \mathrm{O} 605, \lambda 537$ ), à Hector, guerrier dont on a pu montrer qu'il était à l'origine proche d'Arès ${ }^{40}(\Theta 355$, I 238, П 245, $\Phi$ 5), à Diomède, guerrier valeureux, mais dont la personnalité est trouble et qui a un côté négatif et íßpıণ⿰彳亍 $(\mathrm{E} 185, \mathrm{Z} 101, \Theta 111, \Pi 75)^{41}$. Ailleurs, le verbe est appliqué à divers personnages, mais dans un seul passage : Achille dans son acharnement excessif contre Hector $(\Omega 114=135)$, l'esprit de Zeus en fureur $(\Omega 360$ ), le Cyclope Polyphème ( $(350)$, les prétendants de Pénélope ( $\sigma$ 406), Andromaque, qui craint pour la vie d'Hector ( $Z$ 389), ou un incendie qui fait rage $(\mathrm{O} 605)^{42}$. Il est remarquable que, dans plusieurs passages, Athéna, la déesse de l'intelligence et de la "raison" parle de la folie des autres, dont Arès. Dans la majorité des cas, il s'agit d'un accès de folie, souvent guerrière, qui prend un personnage à un moment crucial de l'action. Ici, apparemment rien de pareil; avant l'intrusion de Lycurgue, Dionysos se trouvait paisiblement avec ses nourrices. Sa folie paraît un état, une maladie mentale dont il est constamment victime.

Lycurgue poursuit les nourrices avec un $\beta 0 v \pi \lambda \hat{\eta} \xi$, un aiguillon à bœufs. Le terme est presque un hapax ${ }^{43}$. Lycurgue écarte les loups, mais ce n'est pas un chasseur, l'aiguillon dénote un bouvier. Or l'élevage des bovins est lié aux plaines riches où se trouvent des cités avec des institu-

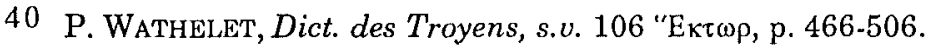

41 P. Wathelet, Dict. des Troyens, s v. 2 "A $\beta \alpha \varsigma$, p. 140-144. Effectivement, aidé par Athéna, Diomède combattra des dieux dans la suite du passage : Aphrodite, Apollon et Arès.

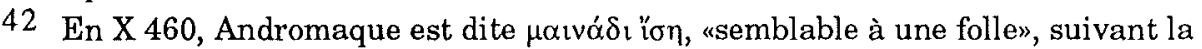

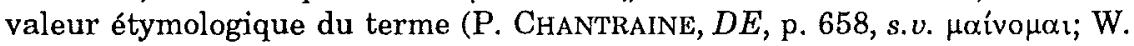
LEAF, The Iliad, 1902², II, p. 462) ou "comme une ménade», si le mot avait déjà pris ce sens. Le jugement des homérologues repose sur l'opinion qu'ils se font de Dionysos chez Homère.

43 I n'apparaît qu'une fois chez HoMÈRE. Il signifie «aiguillon" comme ici ou chez Ps.-Lucien, 82; Philopatris, 4, ou "hache pour abattre un bøuf" comme chez Timon de Phlionte, fr. 4 Diels, P.P.F., cité par Athénée, X, 445d, LÉonidas D'Alexandrie, Anth. Pal., IX, 352, ou Quintus de Smyrne, I, 159. Pour la formation du composé, cf. P. Chantraine, $D E$, p. 917, s.v. $\pi \lambda \dot{j} \sigma \sigma \omega$. 
tions stables. Héra, à laquelle les bovins sont liés, est la déesse de ces institutions et spécialement de celle du mariage ${ }^{44}$. Le fils de Dryas frappe les nourrices du dieu avec un aiguillon, il les traite comme du bétail domestique, même pas comme des êtres sauvages, mais libres.

Dionysos se jette dans la mer. Où que soit la plaine de Nysa, elle se trouve au bord de la mer. Dionysos a des attaches avec la mer ${ }^{45}$. Des récits ou des actes rituels soulignent ce lien. Perpétuel voyageur, Dionysos se trouvait associé à ce qui, pour les Grecs, était le moyen de transport le plus commun, la mer et les vaisseaux qu'elle porte. Se jetant dans la mer, Dionysos allait-il s'y noyer ? Ou bien s'y résorber comme dans une mer primordiale ? Les dieux sont immortels, mais leur immortalité peut être quelque peu oblitérée, comme c'est le cas pour Kronos ou pour les Titans ${ }^{46}$. Affolé, Dionysos sera sauvé par Thétis, la déesse aux pieds d'argent.

Curieuse divinité que Thétis ${ }^{47}$. Fille du vieillard de la mer, déesse marine, elle se présente comme une sorte de divinité primordiale. Telle Métis, elle devait avoir un fils qui serait plus grand que son père. Zeus, qui n'avait pas manqué de tomber amoureux de Thétis, a bien failli y trouver sa perte ${ }^{48}$. Afin d'éviter les effets fâcheux de la destinée, Thétis a dû accepter une mésalliance et s'unir à un mortel. Elle épousera, apparemment sans récrimination, Pélée, qui semble être un homme originel, un homme sorti de la boue $\pi \eta \lambda$ ós $^{49}$. Elle contribue ainsi à établir des liens entre le monde des dieux et celui des hommes. De son côté, Dionysos est fils de Zeus et d'une mortelle.

44 Sur Héra, cf. W. BuRKERT, Greek Religion, p. 131-139, spécialement pour son opposition à Dionysos, p. 133. Les deux divinités se trouvent rapprochées dans plusieurs récits, notamment celui de la folie des Proitides, sans doute parce que, au plan mythique, les contraires s'attirent. Cf. encore M. DETIENNE, Dionysos $\grave{a}$ ciel ouvert, p. 36-38. Dans la version post-homérique du mythe de Lycurgue, l'agressivité du héros est parfois attribuée à Héra (Eumélos, Europia, fr. 11 Bernabé).

45 W. OTto, Dionysos, p. 169-179. Le dieu est lié à l'eau en tant que force vitale primordiale, mais, comme le dieu, l'eau et surtout la mer représentent une force ambiguë, de vie et de mort.

46 Sur les Titans, Hésiode, Théog., 729-739. M.L. WEsT, Hesiod Theogony, p. 336338.

47 W. BURKerT, Greek Religion, p. 172.

48 C'est tout le ressort du Prométhée enchaîné d'Eschyle.

49 P. Wathelet, Le Pélion, $\pi \eta \lambda \hat{\lambda} \zeta$ et Pélée, in Disputationes ad Montium Vocabula ... pertinentes, Vienne, 1969, p. 511-516. 
Du mariage de Thétis et de Pélée allait sortir Achille qui cherchera vainement un équilibre entre ses deux origines, divine et humaine ${ }^{50}$. Mais le mariage en question sera le point de départ d'une autre aventure : l'intervention d'Éris et le jugement de Pâris seront la cause de la guerre de Troie. Comme le racontent les Chants cypriens ${ }^{51}$, la guerre de Troie aura pour but ultime de rétablir un équilibre rompu par le nombre excessif des humains qui écrasaient la Terre.

Dans l'Iliade, Zeus ne peut refuser son aide à Thétis parce que la déesse l'avait tiré d'affaire dans une circonstance spécialement difficile (A 393-406) : il se trouvait seul contre tous les autres dieux. En d'autres circonstances, Thétis avait également sauvé Héphaistos. Le dieu forgeron avait été jeté du ciel par sa mère parce qu'il était boiteux ( $\Sigma$ 394-409).

Thétis apparaît ainsi comme une divinité primordiale, spécialement liée à l'équilibre du monde. Même si l'étymologie de son nom n'est pas assurée, le rapprochement a été fait entre $\Theta \dot{\varepsilon} \tau \iota \varsigma$ et $\tau i \theta \eta \mu \imath^{52}$. Thétis est, de plus, proche de Thémis, déesse des institutions.

Alors que Lycurgue, le civilisateur, le héros de l'ordre des hommes, allait causer la perte de Dionysos, Thétis sauve le dieu et rétablit de la sorte l'équilibre du monde. Le dieu affolé et couard, antithèse vivante des idéaux du monde héroïque, est indispensable à la survie du monde et il est dangereux de le méconnaître.

On comprend dès lors pourquoi Dionysos a fait cadeau à Thétis d'une amphore en or; c'était pour remercier la déesse de son intervention en sa faveur.

Si l'on suit l'Iliade, Lycurgue a été puni de son attitude par Zeus, qui l'a aveuglé, et il n'a guère survécu puisqu'il était un objet de haine pour l'ensemble des dieux. La tradition post-homérique ${ }^{53}$ raconte que c'est

50 P. Wathelet, Les Troyens de l'Iliade. Mythe et Histoire, Paris, Les Belles Lettres, 1989 (Bibl. de la Fac. de Phil. et Lettres de l'Univ. de Liège, 252), p. 155 . 159.

51 Kypria, fr. 1 Bernabé.

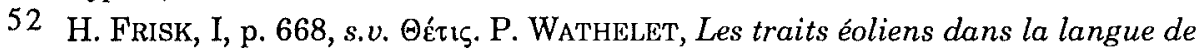
l'épopée grecque, Rome, 1970, p. 106.

53 De la tétralogie que POLYPHRASMON avait composée sur Lycurgue, il ne reste que le titre (TrFG, I, $7 \mathrm{~F} 1$ Snell-Kannicht). On possède quelques fragments des Edoniens d'EschYLE (fr. 57-67 Radt) sur le même sujet. Le dieu lui-même y punissait Lycurgue. Cf. H.J. METTE, Der verlorene Aischylos, Berlin, 1968, p. 136-138, et M. Detienne, Dionysos à ciel ouvert, p. 25-26. Sur l'iconographie suscitée par l'œuvre d'EsCHYLE, importante surtout sur les vases italiotes, cf. 
Dionysos lui-même qui, manifestant sa puissance, a infligé à Lycurgue une punition digne de celle qu'il infligea à Penthée dans les Bacchantes d'Euripide. Devenu fou, Lycurgue prendra son fils Dryas pour une vigne et voulant l'émonder, il lui coupera les extrémités. Ensuite, revenu à la raison, Lycurgue aura conscience de son crime. L'horrible et le macabre le disputent à l'absurde et à la dérision. On ne peut dire si Homère a connu ou non la seconde version. De toute manière, il se serait probablement abstenu de raconter une telle histoire ${ }^{54}$. Ce qui est important c'est qu'il ne montre pas Dionysos en train de se venger luimême, mais qu'il fait exécuter cette vengeance par Zeus et par l'ensemble des dieux. Tout se passe comme si Homère voulait éviter de donner trop de puissance à une divinité dont il se méfie.

Le passage qui nous occupe comporte un certain nombre d'expressions traditionnelles, mais celles-ci sont en nombre limité et surtout elles ne concernent guère Dionysos lui-même. Ainsi, par

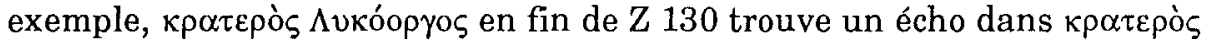

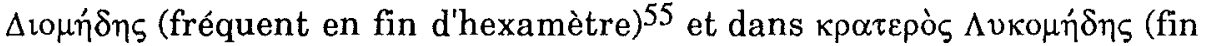

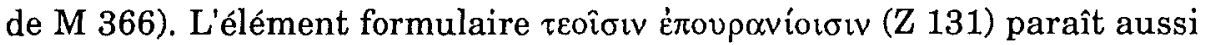
en $Z 129$ et, avec les termes inversés, en $Z 527$. Ti $\theta$ ñv $\alpha \varsigma$ occupe en général

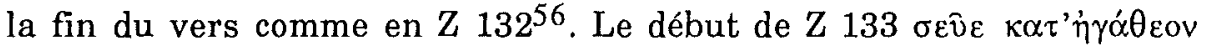
Nvońiov trouve un écho dans le début de Y $189 \sigma \varepsilon \hat{v} \alpha \kappa \alpha \tau$ ' 'I $\delta \alpha i \omega v$. L'expression $\kappa \alpha \tau \dot{\alpha} \kappa \hat{\jmath} \mu \alpha$ occupe la même place qu'en Z 136, en A 483 et

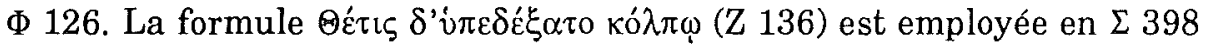

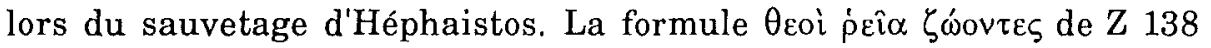
termine aussi $\delta 805$ et $\varepsilon 122$, etc.

L'analyse formulaire du passage montre que celui-ci trouve de nombreux répondants dans le reste de l'œuvre et qu'il participe de la composition traditionnelle comme d'autres morceaux de l'Iliade. Il n'y a donc aucune raison de suspecter son authenticité 57 . En revanche, aucune expression traditionnelle spécialement ancienne ne permet de conclure que le passage serait antérieur à Homère et repris par lui à une tradition archaïque.

A.D. TRENDALL - T.B. WeBSTER, Illustrations of Greek Drama, Londres, 1971, p. 49-52. Cf. aussi Ps.-APOLLOd., Bibl., III, 5, 1 et Hygin, Fab., 132.

54 G. MurRay, The Rise of the Greek Epic, Oxford, 19243, p. 126-128.

$55 \Delta 401,411(=\mathrm{E} 251, \mathrm{~K} 446), \mathrm{E} 143,286(=\Lambda 384), 814, \mathrm{H} 163(=\Psi 290), \Theta 532, \mathrm{~K}$ $369(=\Lambda 361), 536, \Lambda 316,660, \mathrm{M} 366, \Pi 25, \Psi 472,812$.

$56 \mathrm{Z} 389,467, \mathrm{X} 503$.

57 Voir J. VAN LEEUWEN, Ilias cum Prolegomenis, notis criticis, commentariis exegeticis, Leyde, 1912, p. 217; W. LEAF, The Iliad, Londres, 19002, p. 266. 
Il est peut-être un autre personnage qu'il convient de mentionner avec Dionysos, c'est Briséis ${ }^{58}$. On sait que le héros ou les héroïnes de l'épopée sont souvent proches d'une ou de plusieurs divinités, dont ils incarnent en quelque sorte les qualités et les défauts. Comme Bpırús était, à Lesbos ${ }^{59}$, une épithète du dieu et que Briséis a des attaches avec Lesbos ${ }^{60}$, il est possible qu'il faille rapprocher le dieu et l'héroïne. Le rôle de Briséis est capital dans l'Iliade et il n'est pas sans évoquer le côté irrationnel du dieu. La "raison", le bon sens voudrait que les chefs achéens, qui se trouvent aux prises avec un siège interminable, fassent preuve de cohésion entre eux et s'unissent contre la difficulté. Or, c'est tout le contraire qui se produit. L'autoritarisme maladroit d'Agamemnon provoque Achille en lui enlevant Briséis. Il faudra l'intervention soudaine et efficace d'Athéna pour empêcher le fils de Pélée de frapper l'Atride. Provisoirement, l'élément raisonnable, Athena, triomphe des sentiments irrationnels, mais l'enlèvement de Briséis provoquera néanmoins tous les drames que l'on sait, y compris la mort de Patrocle. C'est justement au sujet de Patrocle que Briséis intervient directement. Rendue enfin à Achille, elle fait l'éloge funèbre du héros ${ }^{61}$. C'est l'occasion pour elle de dire qu'elle a espéré qu'Achille l'épouserait. Espoir aussi vain qu'irrationnel.

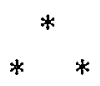

En somme, Homère et Hésiode mentionnent Dionysos à plusieurs reprises, notamment comme dieu du vin. Ils font quelques allusions à la naissance du dieu et à ses rapports avec Ariane, mais sans fournir de précision, ce qui donne à entendre que, dans ses grandes lignes, le récit est bien connu au moins d'une partie du public. L'apparition de Dionysos dans le mythe d'Ariane est d'autant plus remarquable qu'il s'agit d'une héroïne très archaïque. Le seul récit un peu développé qui apparaisse dans l'épopée est l'épisode de Lycurgue raconté par Diomède. L'histoire est exemplaire. Si, dans la bouche de Diomède, elle enseigne

58 P. Wathelet, Dict. des Troyens, s.v. 74 B $\rho$ loni is, p. 367-372.

59 Androtion, 324 F 56 Jacoby, in Etym. Magnum, 214, 5 ( $\beta$ p $6 \alpha i ̂ \varsigma)$ ). STÉPH. Byz., s.u. Bpîo $;$ I.G., XII 2, 484, 1. 2 (décret d'époque impériale à Hiéra en

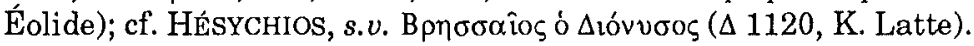

60 Briséis est associée au sept captives venues de Lesbos qu'Agamemnon offre en dédommagement à Achille (I 128-132).

61 T $282-300$. 
qu'il ne faut pas s'attaquer aux dieux, sa portée est plus riche de signification. Il faut éviter l'excès en tout, même dans ce qui, à première vue, paraîtrait raisonnable. On ne peut totalement éliminer Dionysos, le dieu fou, qui incarne précisément tout ce qui s'oppose à la sagesse ou à la raison humaine. La société des hommes ne peut, sans grand péril, ignorer cette part d'irrationnel, qui émane d'elle-même, même si elle lui semble étrangère ou si elle la voudrait telle. Dionysos n'est pas un dieu héroïque, il n'incarne pas les idéaux aristocratiques des héros qui multiplient les hauts faits dans l'épopée. Dans la famille des dieux, il semble le parent mal venu, sans naissance, anormal, que le lignage s'efforce de dissimuler, comme par pudeur. De plus, tel Déméter, c'est une divinité de paysans et non de combattants glorieux. Très marqué par la vie de la terre, Hésiode aurait pu lui réserver un meilleur sort. Toutefois, ici encore, Dionysos n'a aucune chance. Le vieux poète d'Askra est trop épris d'ordre et de respectabilité pour faire une large place à un dieu qui prend, comme à plaisir, le contre-pied de toutes les valeurs reçues. En un mot, si Dionysos n'apparaît qu'occasionnellement dans l'épopée, ce n'est pas parce qu'il est étranger ou récent dans le panthéon grec, mais parce qu'il représente un aspect de la divinité qui ne correspond pas à l'image que les poètes veulent donner des dieux et que, par conséquent, il dérange. Marginal par rapport aux dieux de l'Olympe, Dionysos le dieu fou, homme efféminé, engendre tout à la fois, par ses dons irrationnels, la joie et la peine.

Les traits du dieu révélés par l'analyse de l'épopée sont attestés de manière beaucoup plus explicite dans les siècles qui suivent, que ce soit dans le rituel ou dans la littérature. Il n'est pas question dans les limites de ce bref article d'analyser les innombrables facettes de ce que nous savons de Dionysos à travers les âges. Pour ne pas sortir du domaine littéraire, on se bornera à évoquer brièvement deux œuvres presque contemporaines et qui ont des tonalités différentes puisqu'il s'agit d'une tragédie et d'une comédie, les Bacchantes d'Euripide et les Grenouilles d'Aristophane.

Dieu des enthousiasmes collectifs et aussi de l'illusion, Dionysos patronne le théâtre. Ce dernier constitue une manifestation cultuelle en l'honneur du dieu et, à l'inverse du théâtre médiéval européen, qui est sorti de l'Église, le théâtre grec est resté une manifestation cultuelle, du moins jusqu'à la fin de l'époque classique.

La tragédie des Bacchantes est une des dernières d'Euripide, on la considère souvent comme son chef-d'œuvre. Composée entre 408 et 406 
alors que le poète s'est retiré en Macédoine, elle sera jouée à Athènes après sa mort, en 406 ou peu après ${ }^{62}$.

D'une manière beaucoup plus explicite que dans l'épopée, Dionysos est montré comme un dieu qui se déplace ${ }^{63}$. Son côté irrationnel et ambigu, qui le rapproche d'Aphrodite, en a fait un dieu asianique ${ }^{64}$. Son retour dans sa cité natale, où sa divinité n'est pas reconnue ( Nul n'est prophète en son pays»), marque, en quelque sorte, le triomphe de l'Asie sur la Grèce, comme l'Iliade avait vu le triomphe de la Grèce sur l'Asie, celui d'Athéna sur Aphrodite.

Entouré de Ménades, ayant transformé les femmes de Thèbes en Bacchantes et les ayant fait quitter leurs métiers et leurs fuseaux, occupation typiquement féminine, Dionysos apparaît comme un être efféminé, aux boucles blondes et aux yeux aguicheurs. Il prétend rétablir l'ordre dans la cité, mais son ordre n'est pas celui des sages, ni celui de la raison. Les personnalités les plus fragiles ont cédé à son empire. Les femmes sont retournées à un contact total avec la nature, des vieillards comme Tirésias et Cadmos se sentent rajeunir et, avec un calcul qui n'est pas dissimulé, ils suivent le mouvement général. Les hommes du commun sont sur le point de céder eux aussi, mais le roi, Penthée, gardien intransigeant et conservateur des bonnes mœurs, prétend rétablir une situation rationnelle. Incapable de tout mysticisme, Penthée reprend les explications rationalisantes données jadis par sa mère et par ses tantes sur la naissance de Dionysos. Dans les transports des Bacchantes, il ne voit qu'une manière de dissimuler les pires désordres sexuels. Pour lui, Tirésias n'entre dans le jeu du dieu que pour de l'argent, le vin doit rester l'unique apanage des hommes. La raison raisonnante conforte chez lui l'égoïsme masculin.

Ce qui déroute Penthée c'est que Dionysos n'a aucun des caractères habituels des dieux. Efféminé, il oppose au roi imbu de son pouvoir, autant que le Créon de l'Antigone de Sophocle, la douceur et la passivité. Veut-on l'enchaîner, il se laisse enchaîner, mais les chaînes tombent d'elles-mêmes. Veut-on lui opposer des soldats, leurs armes se révèlent sans force. Au sérieux inquiet de Penthée, il oppose sa douceur et son sourire. C'est un dieu pacifique qui répartit les biens entre riches et pauvres.

62 P.E. Easterling - B.M.W. KNox, Greek Literature, Cambridge Univ. Press, 1985 , p. 316, 323-324.

63 W.F. OTTO, Dionysos, p. 86-92.

64 Bacch., 64. 
Dionysos représente un dieu qui rejette toutes les valeurs réputées sûres chez les humains. Il en montre la vanité. Il inspire des sentiments irrationnels et provoque des miracles. Néanmoins, sa douceur n'est que trompeuse apparence et le dieu n'a ni pitié, ni miséricorde. Sa puissance éclate d'autant plus qu'on ne s'y attendait pas. Les transports des femmes de Thèbes constituaient déjà une punition ${ }^{65}$. Celle qu'il infligera à Penthée, dont il souligne que son nom a un caractère sinistre ${ }^{66}$, sera effroyable. L'austère rigorisme du roi et son rationalisme dissimulaient des sentiments moins avouables. Déjà son orgueil l'avait amené à s'en prendre sottement aux instruments qu'utilisait Tirésias pour ses oracles : il ne se borne pas à nier la divinité de Dionysos, il attaque aussi Apollon. Croyant que les Bacchantes se livrent à des orgies indécentes malgré le récit que lui a fait un de ses bouviers, Penthée accepte de suivre Dionysos afin d'aller les espionner. Son goût de voyeur causera sa perte. Subjugué par le dieu dont il revêt la livrée, il s'offre lui-même à une mort atroce. Les hommes et les femmes qui se flattaient de leur sagesse sont à leur tour pris de folie et le dieu triomphe de la manière la plus totale, même si Agavé, en digne héroïne d'Euripide, s'interroge sur la cruauté impitoyable du dieu.

Dans la tragédie, la présentation peu flatteuse de Dionysos est compensée par sa victoire finale. Comme Lycurgue chez Homère, Penthée n'a pas reconnu la divinité de Dionysos. Ce dernier triomphe.

La situation est quelque peu différente si on se tourne vers les Grenouilles d'Aristophane ${ }^{67}$.

Nous sommes à Athènes en 405, la situation politique et militaire est des plus graves, les Athéniens sont étreints par une légitime angoisse. Les temps ne sont plus à attaquer directement tel politicien en vue, le sujet ne saurait plus être traité avec la sérénité voulue, aussi Aristophane préfère-t-il reprendre un sujet littéraire : Euripide et Sophocle viennent de mourir, Agathon a quitté Athènes ${ }^{68}$, les poètes tragiques qui suivent ne sont pas, d'après le Comique, dignes de leurs aînés. Dans une comédie spécialement riche en trouvailles et en rebon-

65 On trouve un thème analogue à Argos. Tantôt, les filles de Proïtos refusent de recevoir les rites de Dionysos (Corpus hésiodique, fr. 131), tantôt elles insultent une statue d'Héra (AKousilaos, 2 F 28 Jacoby).

66 A. FICK - F. BECHTEL 2 , p. 406.

67 P.E. EAsterling - B.M.W. KNox, Greek Literature, p. 376. W.B. STANFORd, Aristophanes Frogs, Bristol Classical Press, 1958.

68 P.E. EAsterling - B.M.W. KNox, Greek Literature, p. 341. 
dissements, comme si le rythme endiablé des gags devait répondre à la tension nerveuse des spectateurs, Aristophane montre Dionysos qui décide de descendre aux Enfers, comme l'avait fait Héraklès, mais cette fois pour aller rechercher le meilleur des poètes tragiques.

Le dieu est l'élément dynamique et ridicule de la comédie. Vêtu d'une robe de femme, sur laquelle il a placé la peau de lion et la massue caractéristiques d'Héraklès, il ne cesse d'étaler sa vantardise et sa couardise. D'une instabilité d'humeur indigne d'un homme, il incarne tous les défauts possibles. À un moment où Xanthos prétend qu'une Empouse vient d'apparaître, Dionysos affolé descend précipitamment de l'estrade et se jette dans les bras de son prêtre qui siège au premier rang des notables. Rompant, comme souvent, la convention théâtrale, Aristophane provoque le rire en montrant un monde à l'envers : c'est le dieu qui demande protection au prêtre qui devrait normalement trouver en lui son appui.

Le même renversement des valeurs établies caractérise toute la pièce. Instable, Dionysos ne cesse d'être dupé par son esclave Xanthias, qui annonce dans une certaine mesure le Scapin de Molière. Franchissant l'Achéron en barque, c'est lui qui ramera à la place de Charon, mais sous sa direction. Sa qualité de dieu ne l'empêchera pas de se disputer avec les grenouilles, qui donnent son titre à la pièce, on est loin d'Orphée qui charmait les animaux. Dernière dérision du passage, alors que Dionysos rame avec effort pour traverser le marais infernal, Xanthias en fait le tour à pied paisiblement et beaucoup plus commodément : il arrive de l'autre côté avant son maître ${ }^{69}$. L'entrée dans le domaine d'Hadès n'est pas moins riche en facéties qui se terminent par un concours d'endurance au fouet pour décider qui des deux personnages est effectivement le dieu. L'épisode brocarde allègrement la cérémonie, bien connue à Sparte, de la flagellation des jeunes gens en l'honneur d'Artémis Orthia. Étrange dieu que Dionysos qui n'est pas reconnu et qui se fait flageller. Entré enfin dans la demeure d'Hadès, Dionysos continue son rôle de bouffon paradoxal. Amené à arbitrer le conflit entre Eschyle et Euripide, il est incapable d'assurer sereinement son rôle de juge, mais coupe le débat de réflexions et de plaisanteries terre à terre qui devaient amuser le petit peuple d'Athènes.

La comédie multiplie à plaisir les traits opposés à l'opinion qu'on se fait en général des dieux : bien qu'immortel, Dionysos s'ennuie telle-

69 P. Wathelet, Le retour de Néoptolème et les Grenouilles d'Aristophane, in Kentron, 4, 2 (1988), p. 27-31. 
ment au théâtre qu'il en sort chaque fois vieilli d'un an ${ }^{70}$. Sa peur est telle qu'il souille sa robe ${ }^{71}$. Il est appelé "fils de cruchon»72. Couard, vantard, efféminé, instable, il est aussi gourmand et lubrique.

Certes la comédie ancienne ne respecte rien. Comme les autres auteurs comiques, Aristophane brocarde allègrement tout ce qui est respectable et avec une liberté presque totale. La tragédie évite de mettre en scène les grandes divinités et, quand elle le fait, souvent dans des prologues ou dans la scène finale de la pièce (le célèbre deus ex machina), la divinité est traitée avec respect, elle apparaît avec le caractère et les attributs que lui confère en général la tradition. Nous connaissons malheureusement très mal les comédies qui mettent en scène des divinités et notamment Zeus ${ }^{73}$, mais, pour autant que nous puissions en juger, elles donnent des dieux une vue caricaturale sans doute, mais qui respecte leurs qualités traditionnelles. Dans les Grenouilles, Aristophane affuble Dionysos de traits qui sont tout à l'opposé de ce qu'on prête en général aux dieux. Le fait est d'autant plus remarquable que, comme on le rappelait plus haut, la comédie est partie intégrante du culte de Dionysos. On ne peut invoquer un relâchement général de la croyance religieuse, puisque la même époque voit des procès d'impiété retentissants, celui de Protagoras en $411^{74}$ et celui de Socrate en 399. La seule solution au problème - la brève analyse des Bacchantes l'a déjà suggéré - est que le Dionysos des Grenouilles est, en gros, conforme à la représentation qu'on s'en faisait au Ve s. : comme le dieu fou de l'Iliade, le Dionysos d'Aristophane incarne des qualités irrationnelles, opposées à toutes celles qu'on révère chez d'autres divinités.

Ainsi s'explique le paradoxe apparent d'un dieu qui est ridiculisé dans son culte même et par ses dévôts. L'explication tient peut-être dans l'appréhension même du sacré qui, par nature, échappe à la raison et surtout ne se laisse pas envelopper par elle. Le royaume du dieu n'est pas de ce monde car on ne peut en connaître les limites. Le dieu est à ce point fort qu'il n'a que faire des jugements et des valeurs humains, rien ne lui est impossible même de se nier ou de se détruire, folie pour les hommes, sagesse pour la divinité. Les hommes qui incarnent la raison

70 Gren., 17-18.

71 Gren., 308.

72 Gren., 22.

73 Voir toutefois la Némésis de Cratinos, fr. 114-117 (R. Kassel - C. Austin, PCG, IV, p. 179-185).

74 E. DERENNE, Les procès d'impiété, Liège, 1930, p. 45-55. 
ou la puissance et prétendent être possesseurs du sacré sont en réalité pris de folie et, comme Penthée, ils sont déchirés par les Bacchantes; ailleurs, ils seront traités de "race de vipères" et de "sépulcres blanchis» et «la vigne qui leur avait été confiée leur sera enlevée».

$\mathrm{Si}$, par son caractère participationiste et par le renversement des valeurs qu'elle contient, la religion de Dionysos évoque le christianisme, il est un point sur lequel elle s'y oppose radicalement : c'est sa violence sans pitié. Toujours en marche, illustrant les caractères les plus opposés à l'idéologie des sociétés en place, Dionysos fait preuve d'une violence impitoyable autant qu'égoïste. Dans les Bacchantes, la punition infligée à Penthée, à Agavé et même à Kadmos est sans rémission.

Dieu inquiétant par ce que sa folie peut avoir d'inattendu et de violent, Dionysos règne sur les mouvements de foule dépourvus de raison. Il représente une réalité psychologique et sociale que les gouvernants ne peuvent méconnaître, dont ils doivent tenir compte et qu'ils s'efforcent d'endiguer sans jamais y parvenir complètement. La folie en la matière serait de ne pas faire la part du feu, de vouloir, comme le législateur Lycurgue, imposer un ordre et une raison à ce qui est par nature désordonné et irrationnel. De même que Thétis, gardienne de l'équilibre du monde, a eu la précaution de sauver Dionysos, de même, les sociétés antiques avaient la sagesse d'admettre en leur sein et à des moments précis des périodes de folie collective qui n'épargnaient personne, mais que l'on tentait de canaliser avec plus ou moins de succès. Les drames satyriques et la comédie ancienne ont en quelque sorte institutionalisé le courant dionysiaque. Dans la Chrétienté du moyen âge, les fêtes des fous et les carnavals constituaient une manière de soupape de sécurité qui libéraient les sociétés de pulsions irrationnelles. Les crises religieuses du XVIe s., le jansénisme et l'arrivée au pouvoir de la bourgeoisie ont eu pour effet d'éliminer de telles manifestations, jugées déplacées. Le résultat de cette imprévoyance se mesure de nos jours dans les stades de football et, si la violence y était jugulée, elle risquerait de se trouver un autre exutoire.

En somme, le Dionysos de l'épopée est, pour l'essentiel, le même que celui de la période classique. C'est le dieu du monde à l'envers, qui prend systématiquement la position opposée à tout ce qu'on révère, ou que la raison ferait attendre. Sorte de dieu fou et redoutable, il incarne le contre-pied des idéaux de la société humaine. Dans un monde masculin qui cultive la stabilité et les vertus guerrières, Dionysos se présente comme un homme-femme, toujours en voyage, couard et aussi peu guer- 
rier que possible. Fils d'une mortelle, il brouille toutes les hiérarchies; adulte-enfant entouré de plusieurs nourrices, il fuit à la première alarme et, bien qu'immortel, il disparaîtrait si Thétis ne le sauvait; il est le dieu fou; il est associé à Ariane pour la faire périr; il donne le vin, mais aussi la mort.

On sait qu'Homère évite ce qui est trop marqué par la religion ou la transcendance; son propre goût pouvait l'écarter d'un dieu dont le culte est hautement participatif. De plus, Dionysos pouvait, comme Déméter, passer surtout pour un dieu d'agriculteurs.

La société des auditeurs d'Homère a des idéaux aristocratiques et elle se trouve en crise : la croissance démographique a entraîné une surpopulation dont le trop-plein va aller fonder des colonies. L'arrivée à l'âge adulte de nouvelles couches de population crée un problème d'adaptation de ces jeunes aux vieilles structures : le héros principal de l'Iliade, Achille est un jeune mal adapté et Ulysse, le héros de l'Odyssée, explore des terres nouvelles. Dans une telle situation, il est peu opportun et peu recommandable de faire l'apologie d'un dieu qui, par nature, ébranle toutes les institutions. C'est là sans doute la raison de la discrétion des aèdes vis-à-vis de Dionysos. Ils connaissent le dieu et leur auditoire aussi puisqu'ils se contentent d'allusions peu explicites, mais ils préfêrent ne pas lui donner trop de place car il pourrait être de mauvais exemple.

À l'inverse de l'épopée, le théâtre attique du Ve s. a fait une large place à Dionysos. La société athénienne du Ve s. est ou se croit forte et solide. Elle tolère largement sa propre contradiction. La comédie ancienne brocarde et se moque des personnages les plus importants de l'État, sans que, pour la cause, celui-ci s'en trouve ébranlé. Plus tard, à la fin du siècle et au IVe, la situation politique empire et les auteurs comiques devront se montrer plus prudents et mettre une sourdine à leurs attaques personnelles.

Divinité de l'irrationnel, du monde à l'envers, Dionysos est honoré en tant que tel dans une cité qui a la sagesse de ne pas faire comme Lycurgue et de tolérer en son sein une part d'irrationnel, comme il y en a toujours une dans l'homme et que l'on ne peut jamais complètement éliminer. Mais la prudence de la cité grecque va plus loin. En accueillant Dionysos et son culte, elle s'est efforcée de "civiliser» quelque peu le dieu, dont le culte a perdu de sa sauvagerie primitive, qu'il conserve intacte ailleurs, pour s'intégrer dans un cadre institutionnel. Loin 
d'être des manifestations désordonnées, les concours dramatiques ont leurs règles, scrupuleusement respectées, même si le dédicataire de la fête reste le dieu qui échappe à toute règle.

Université de Liège,

Paul WATHELET

Place du XX-Août, 32

B - 4000 LIÈGE 